Disponível em

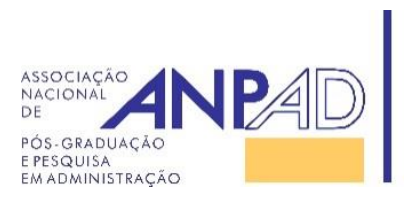

http://www.anpad.org.br/rac

RAC, Rio de Janeiro, v. 20, n. 6, art. 3, pp. 693-714, Nov./Dez. 2016

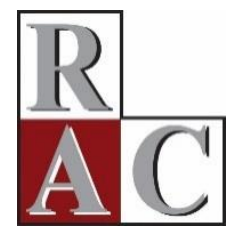

$(\mathrm{oc})$ EY

\title{
O Sotaque Estadunidense Representa uma Vantagem em Decisões de Emprego no Brasil?
}

Is the American Accent an Advantage in Employment Decisions in Brazil?

Bruno Felix von Borell de Araujo ${ }^{1}$

Fabricia Correa ${ }^{1}$

Mark Wolters ${ }^{2}$

Fundação Instituto Capixaba de Pesquisas em Contabilidade, Economia e Finanças ${ }^{1}$ University of Illinois ${ }^{2}$

Artigo recebido em 07.07.2015. Última versão recebida em 20.10.2015. Aprovado em 26.10.2015. Publicado online em 26.08.2016. 


\title{
Resumo
}

O objetivo deste estudo foi examinar a relação entre o sotaque de um candidato a uma vaga de trabalho hipotética, bem como suas características pessoais (competência percebida e cordialidade), e os julgamentos relacionados ao desempenho deste no trabalho pretendido (adequação ao cargo, probabilidade de promoção e de contratação). Para tal, foi realizado um experimento com uma amostra de 304 alunos e ex-alunos de um curso de MBA em Gestão de Pessoas, de uma instituição de ensino superior sediada na cidade de São Paulo. Os resultados mostraram que, comparados a um candidato a uma vaga de trabalho brasileiro, o profissional com um sotaque estadunidense no idioma português foi mais bem avaliado em termos de adequação ao cargo, probabilidade de contratação e competência percebida. O candidato brasileiro, por sua vez, recebeu avaliações mais favoráveis em termos de probabilidade de promoção e cordialidade percebida. Uma vez que foram identificados vieses nos julgamentos relacionados ao trabalho realizados pelos participantes da pesquisa, sugere-se que as empresas busquem contratar selecionadores menos propensos a julgamentos estereotipados de candidatos a vagas de trabalho.

Palavras-chave: seleção; estigma; sotaque; estrangeirismo; decisões de emprego.

\begin{abstract}
The aim of the study was to examine the relationship between the accent of hypothetical job candidates and personal characteristics of the candidates (perceived competence and warmth) with job related decisions (job suitability, likelihood of promotion and likelihood of hiring). For this purpose, an experiment was conducted with a sample of 304 students and alumni of an MBA course in Personnel Management from an institution of higher education based in São Paulo. The results showed that candidates with an American accent in Portuguese were evaluated better in terms of suitability for the position, likelihood of hiring and perceived competence than candidates with a Brazilian accent. The Brazilian candidate, in turn, received more favorable ratings in terms of probability of promotion and perceived warmth. Since we identified hiring biases, we suggest that firms should seek out human resource employees that are less likely to use stereotypical judgments in their evaluation of job candidates.
\end{abstract}

Key words: selection; stigma; accent; foreignness; employment decisions. 


\section{Introdução}

O mercado de trabalho contemporâneo tem sido cenário de frequentes mobilidades de profissionais para economias mais favoráveis em termos de remuneração e oportunidades de carreira (Almeida, Fernando, \& Sheridan, 2012). O Brasil, diante da crise financeira global, tem sido apontado frequentemente como um dos destinos mais procurados para profissionais que buscam se recolocar no mercado global (Araujo, Teixeira, Cruz, \& Malini, 2014; Brookfield Global Relocation Services, 2012; G. G. Reis, Fleury, Fleury, \& Zambaldi, 2015). Sendo assim, diversos estrangeiros têm disputado vagas no mercado laboral brasileiro com profissionais locais, o que aumenta a complexidade dos processos seletivos envolvendo essa diversidade de profissionais (Coordenação Geral de Imigração [CGIg], 2012). Tal fato faz com que as seleções de pessoal tornem-se mais suscetíveis a julgamentos não racionais e baseados em estigmas ou favorecimentos ao candidato estrangeiro a partir de características pessoais desses trabalhadores como, por exemplo, o sotaque (Stone-Romero \& Stone, 2007).

Nesse contexto global de pluralidade cultural entre candidatos a postos de trabalho, estudos anteriores têm buscado comparar a influência de diferentes sotaques de candidatos nos julgamentos de selecionadores de vagas de trabalho (Pudelko, Tenzer, \& Harzing, 2014). O estudo de Singer e Eder (1989) investigou o impacto do sotaque de chineses, alemães e neozelandeses em decisões de emprego realizadas por neozelandeses e identificou que esses candidatos foram preteridos em relação a locais. Resultados semelhantes foram identificados por Lev-Ari e Keysar (2010), que reportaram o achado de que o sotaque de poloneses, turcos, austro-germânicos, coreanos e italianos para o inglês foi avaliado como menos confiável que o de estadunidenses por avaliadores nativos dos Estados Unidos. Mais recentemente, Hosoda, Nguyen e Stone-Romero (2012) identificaram indícios de que profissionais com sotaque hispânico tendem a enfrentar maiores dificuldades ao ingressarem no mercado norte-americano do que aqueles que possuem um sotaque típico de um nativo dos Estados Unidos para o idioma inglês. Entretanto, nem todas as pesquisas apontam desfavorecimentos a estrangeiros: Hosoda e Stone-Romero (2010) identificaram em seu estudo que candidatos com sotaque francês receberam avaliações mais positivas que aqueles que apresentaram sotaque japonês ou estadunidense ao falarem o idioma inglês.

Os citados estudos apresentam a característica dominante de discutir a discriminação a candidatos estrangeiros em países de língua inglesa. Apesar da inegável contribuição de tais estudos, observa-se uma lacuna na literatura, no sentido de melhor compreender a influência do sotaque de profissionais estrangeiros em outros ambientes profissionais de destino, além dos tradicionalmente pesquisados países de língua inglesa (Offermann, Matos, \& Basu DeGraaf, 2014). Além disso, nota-se uma oportunidade também de aprofundar as pesquisas em casos nos quais a figura do estrangeiro possa ser associada a imagens mais positivas que a de candidatos nacionais. Dado o crescimento do Brasil como ambiente hospedeiro de trabalhadores de diferentes origens - uma vez que a maioria dos estrangeiros inseridos no mercado de trabalho brasileiro são estadunidenses (CGIg, 2012), e que profissionais oriundos dos Estados Unidos costumam receber julgamentos de supervalorização no contexto laboral brasileiro (Motta, Alcadipani, \& Bresler, 2001), seria relevante para a literatura explorar empiricamente se variações no sotaque do português falado por brasileiros e estadunidenses representam diferenças em termos de possibilidade de inserção e progressão no mercado de trabalho.

Visando preencher essa lacuna na literatura, o objetivo deste estudo foi examinar a relação entre o sotaque de um candidato a uma vaga de trabalho hipotética e julgamentos relacionados ao trabalho (adequação ao cargo, probabilidade de promoção e de contratação) e características pessoais dos candidatos (competência percebida e cordialidade). Para tal, foi realizado um experimento com uma amostra de 304 alunos e ex-alunos de um curso de MBA em Gestão de Pessoas de uma instituição de ensino superior sediada na cidade de São Paulo.

O presente estudo busca tanto oferecer contribuições acadêmicas como práticas. Ele contribui para a literatura sobre Gestão de Recursos Humanos no sentido de avaliar possíveis vantagens ou desvantagens que profissionais oriundos de um país economicamente dominante enfrentam em um mercado hospedeiro emergente. Em termos práticos, provê informações a selecionadores a respeito de 
dados relativos a quanto o julgamento de estrangeiros pode ser influenciado por vieses que podem afetar a qualidade da seleção.

\section{Referencial Teórico}

Neste tópico, desenvolve-se uma revisão da literatura, na qual inicialmente apresentam-se as duas correntes de estudo mais recorrentes para a investigação da influência do sotaque de candidatos em decisões de emprego (correntes instrumental e simbólica). Nesta última, desenvolve-se o argumento que norteia o presente estudo: selecionadores não utilizam modelos plenamente racionais de decisão em processos seletivos, mas sim modelos considerados plenamente satisfatórios. O Modelo de Eliminação Sequencial (Gati, 1986) para a tomada de decisões foi a perspectiva adotada para fundamentar essa ideia. O argumento do estudo também sustenta que o sotaque é um dos elementos simbólicos que são usados por selecionadores de pessoas para realizar julgamentos simplificados (que visam ser satisfatórios, e não plenamente racionais) e especulativos a respeito de candidatos em processos seletivos de trabalho. Para sustentar essa ideia, adotou-se o modelo de Estigmatização nas Organizações, de Stone-Romero e Stone (2007), que se encontra alicerçado em Goffman (1963). Por fim, o argumento também sustenta que a estigmatização pode se dar não somente por desvantagens, mas também por favorecimentos ao candidato portador de um sotaque estrangeiro para o idioma falado no país no qual busca se colocar profissionalmente. Para alicerçar o estudo desse favorecimento a determinados candidatos estrangeiros (neste caso, estadunidenses) no contexto brasileiro, foi abordado a conceito do traço cultural nacional do Estrangeirismo (Calligaris, 1991; Holanda, 1995).

Sendo assim, após a apresentação das correntes de estudo sobre a influência de sotaque em decisões de emprego, apresentam-se neste tópico os modelos teóricos da Eliminação Sequencial e de Estigmatização nas Organizações, assim como a fundamentação conceitual para o Estrangeirismo. A seção é finalizada com a sustentação e a apresentação das hipóteses testadas no estudo.

\section{Correntes para o estudo da influência do sotaque em decisões de emprego}

O sotaque de uma pessoa pode afetar o julgamento a seu respeito por duas razões principais: (a) Instrumental: $\mathrm{O}$ sotaque do emissor de uma mensagem dificulta a compreensão por parte de quem a recebe e, portanto, pode dificultar a comunicação; e (b) Simbólico: O sotaque possui um efeito de sinalização e, por conseguinte, representa um significado socialmente compartilhado (Ferguson \& Zayas, 2009; Lev-Ari \& Keysar, 2010).

A perspectiva instrumental se baseia na ideia de que o sotaque em si apresenta características inerentes, as quais dificultam a comunicação e, por isso, representa uma desvantagem em interações sociais. Para Oppenheimer (2008), a facilidade com que se pode processar o estímulo linguístico, ou seja, a fluência da comunicação (Schwarz, 2004) afeta o julgamento que as pessoas fazem umas das outras. Estímulos linguísticos mais facilmente processados são considerados como mais prazerosos em termos de escuta (Reber, Schwarz, \& Winkielman, 2004), e isso significa que o receptor da mensagem desfruta de um estado de maior relaxamento e gozo quando interage com um possuidor de um sotaque de fácil assimilação, o que pode afetar positivamente o julgamento feito sobre a pessoa com quem se comunica. Comunicações mais facilmente compreendidas também são avaliadas como menos arriscadas ou sujeitas a desentendimentos (Song \& Schwarz, 2009) e, consequentemente, mais confiáveis (Reber \& Schwarz, 1999). A redução da incerteza provocada pela clareza da comunicação aumenta a previsibilidade e as chances de os interlocutores se julgarem de maneira mais positiva (Mcglone \& Tofighbakhsh, 2000; Schwarz, Sanna, Skurnik, \& Yoon, 2007; Unkelbach, 2007). Sendo assim, possíveis desvantagens enfrentadas por estrangeiros em processos seletivos poderiam ser vistas como resultados de uma avaliação racional, lógica e instrumental,

Por outro lado, a segunda perspectiva citada por Lev-Ari e Keysar (2010) — a de o sotaque possuir um efeito simbólico - se baseia na ideia de que o sotaque, como dimensão das características do 
discurso de indivíduos, é um elemento que permite associações especulativas (e, portanto, dotadas de racionalidade limitada) a características não visíveis dos interlocutores. Entre essas características, podese destacar o status econômico-social, a etnia e a nacionalidade, e o nível educacional da pessoa que se comunica com determinado sotaque (Singer \& Eder, 1989; p.e. Hosoda, Nguyen, \& Stone-Romero, 2012; Lick \& Johnson, 2013). Assim, nesta perspectiva, o indivíduo como portador de sotaque tende a ser visto com um membro de um grupo externo e, portanto, encontra-se mais sujeito a julgamentos baseados em especulações (Fazio, Barber, Rajaram, Ornstein, \& Marsh, 2013). A seguir apresentam-se as três abordagens teóricas que fundamentam o argumento deste estudo.

\section{O modelo de eliminação sequencial de tomada de decisões}

Os modelos racionais de tomada de decisão tradicionalmente propunham que indivíduos buscam alcançar ganhos ótimos ao multiplicarem a probabilidade e o valor percebido de diferentes opções de decisão e selecionando a que apresenta maior valor. Esse modelo de decisão, baseado na teoria da utilidade esperada (Neumann \& Morgenstern, 1953), possui a característica de ser sequencial, sistemático e científico (Gellat, 1962), e deve considerar todas as possibilidades de ação disponíveis (Katz, 1966).

Neste estudo, defendemos que esse modelo de decisão não é o que se faz presente em processos seletivos de pessoal. Gati (1986) afirma que as pessoas frequentemente não consideram todas as opções disponíveis para uma decisão ou falham ao acessar as probabilidades de eventos. Ele também destaca as limitações que indivíduos possuem ao processar cognitivamente todas as opções disponíveis para uma decisão. Baseado na ideia de que as pessoas não optam por decisões ótimas, mas suficientemente satisfatórias (Simon, 1955; Soares \& Barbedo, 2013), esse autor defendeu que o processo decisório envolve uma eliminação prematura de opções que aparentemente não satisfazem os requisitos dos resultados desejados. A proposta teórica de Gati (1986), conhecida como Modelo de Eliminação Sequencial, sugere que os indivíduos buscam modelos de tomada de decisão mais simples, baseados em experiências passadas e que não envolvam cálculos exaustivos, o que se aplica especialmente a decisões complexas e que envolvem subjetividade.

Embora esse modelo esteja sujeito a julgamentos baseados em emoções, preconceito, discriminação e outros fatores que diminuem a racionalidade das decisões, as pessoas tendem a utilizálo em razão do fato de ele apresentar um melhor custo/benefício para decisões rotineiras e que envolvam maior subjetividade (Murtagh, Lopes, \& Lyon, 2011). As decisões de contratação e promoção de pessoas em organizações - por se basearem em características individuais subjetivas como competência e cordialidade percebidas e estarem sujeitas a preconceitos - podem ser apontadas como exemplos passíveis de ilustrar a lógica de racionalidade limitada na tomada de decisões proposta no Modelo de Eliminação Sequencial (Hosoda et al., 2012).

\section{O modelo de estigmatização social}

O modelo de estigmatização nas organizações, de Stone-Romero e Stone (2007), alicerçado em Goffman (1963), é uma lente teórica útil para entender a corrente simbólica de estudos sobre a relação entre sotaque e decisões de emprego. Nessa perspectiva, um estigma é conceituado como "uma real ou uma discrepância de descrédito profundamente percebida entre as identidades sociais reais e virtuais da pessoa" (Stone-Romero \& Stone, 2007, p. 129). A identidade social virtual (ISV) de um indivíduo representa as expectativas a seu respeito em termo de atributos, tais como habilidades, personalidade, aparência física, atitudes, comportamentos e dicção, em um sistema social. Já a identidade social real (ISR) representa a forma como uma pessoa é capaz de ser percebida ou percebida de fato por um observador (Dubar, 2002; Goffman, 1963).

Dentro do contexto de um processo seletivo, a ISV representa o esperado de um candidato ideal, enquanto a ISR expressa como um indivíduo é verdadeiramente percebido. Sendo assim, um candidato com um sotaque estrangeiro indesejável socialmente pode ser interpretado como não cumpridor dos atributos requeridos para um candidato ideal. Nesse caso, pode haver uma diferença negativa entre a 
ISR e a ISV, o que tende a resultar em uma avaliação estigmatizada e desfavorável ao candidato estigmatizado. Assim, o sotaque estrangeiro pode ser considerado como base para um estigma, resultando, então, em desvantagens para os candidatos nas decisões de contratação (p.e. Hosoda et al., 2012).

O candidato com um sotaque que remete a um grupo social visto como menos dominante tende a ser privilegiado ou prejudicado em decisões de seleção, dependendo do cargo em questão (p.e. Hosoda et al., 2012). Muitas vezes, membros de grupos não dominantes são percebidos pelo grupo dominante como menos adequados para funções de status elevado, como resultado da discrepância negativa entre a ISR e a ISV. Em contrapartida, esses indivíduos podem ser percebidos como adequados para funções de baixo status.

Uma possível explicação para esse dado é o fato de, muitas vezes, as pessoas com sotaque de grupos dominantes serem percebidas como tendo status e poder, enquanto são percebidas com baixos níveis de sucesso socioeconômico as pessoas que possuem perfis de grupos menos dominantes (Cargile, 2000). De acordo com essa premissa, pode-se observar que nos quesitos como competência e inteligência, atributos desejados em cargos de elevado status social, os indivíduos possuidores de sotaques de grupos dominantes tendem a receber avaliações mais positivas do que os de grupos não dominantes (Giles, Willians, Mackie, \& Rosselli, 1995). No entanto, no tocante aos quesitos bondade, cordialidade e solidariedade - características mais requisitadas em cargos de baixo status, indivíduos com sotaques que remetem a grupos não dominantes tendem a ser avaliados de forma mais positiva (Cargile \& Bradac, 2001).

No entanto, a pesquisa de Hosoda e Stone-Romero (2010) aponta uma nova oportunidade de pesquisa ainda pouco explorada: como são avaliados os sotaques de indivíduos provenientes de países que não possuem um estereótipo negativo na cultura de destino? Como Japão e França são países economicamente prósperos, e indivíduos nascidos nesses países são, em geral, respeitados socialmente entre estadunidenses (Deprez-Sims \& Morris, 2010; Hosoda \& Stone-Romero, 2010), os autores realizaram um estudo que comparou avaliações realizadas para o sotaque estadunidense, japonês e francês no idioma inglês. Os resultados mostraram que o candidato com sotaque nativo estadunidense foi mais bem avaliado por estudantes nativos dos Estados Unidos que aquele com sotaque japonês, porém mais mal avaliado que o candidato com sotaque francês. O presente estudo se insere na linha de pesquisa explorada por essa investigação. Optou-se aqui por comparar os julgamentos realizados por brasileiros a respeito de decisões de emprego e características pessoais de candidatos hipotéticos com sotaques brasileiro e estadunidense no idioma português. Com esse objetivo em vista, buscou-se analisar brevemente no tópico seguinte alguns elementos acerca do que se conhece academicamente sobre as relações de trabalho de indivíduos oriundos dos Estados Unidos no Brasil.

\section{Estrangeirismo e o favorecimento a estadunidenses}

O estrangeirismo é um traço cultural brasileiro que pode ser usado para sustentar a hipótese de que estadunidenses teriam vantagens em relação a brasileiros em processos seletivos realizados no Brasil. O estrangeirismo é a valorização do que não é nacional e o menosprezo do que se refere à sua própria nação (Motta et al., 2001). No comportamento dos brasileiros, a admiração e a identificação com a figura do estrangeiro e a negação da brasilidade são elementos recorrentes (Calligaris, 1991; Holanda, 1995). Para Motta, Alcadipani e Bresler (2001), a colonização brasileira - caracterizada pela exploração predatória das riquezas naturais para a comercialização em mercados mais lucrativos - e a miscigenação entre culturas parecem ter contribuído para o desenvolvimento de uma ausência de referenciais internos e o desenvolvimento de uma visão da figura de um estrangeiro superior. Os resultados financeiros das explorações eram dirigidos a uma parcela reduzida da população, formada por europeus e seus descendentes, a qual era constituída por indivíduos que possuíam desprezo pela nação que se formava (Holanda, 1995; Motta et al., 2001). Esse cenário contribuiu para o desenvolvimento de um povo nostálgico por lideranças e heróis platônicos, com condições de vida não adequadas e com alta distância de poder e de posse de recursos financeiros entre as classes sociais. 
Nesse contexto, o sistema de regras e normas sociais adotado no país foi importado de Portugal e pouco ajustado ao contexto brasileiro (Holanda, 1995; Smith et al., 2012). Posteriormente, após a declaração da Independência, seguiu-se um processo de identificação com culturas europeias. Em seguida, a Grã-Bretanha substituiu Portugal na condição de referência cultural e em termos de economia. A posteriori, a França assumiu essa posição, até que os Estados Unidos ocuparam tal posto de referência a partir da Segunda Guerra Mundial (Araujo et al., 2014; Motta et al., 2001). Desde então, no contexto empresarial, as relações de brasileiros com os Estados Unidos têm sido caracterizadas predominantemente pela admiração, explicada pelo estrangeirismo (Bandeira, 2004) e manifestada com base em uma reprodução dos referenciais de gestão oriundos do país norte-americano. Neste estudo, são testadas hipóteses que avaliam se essa relação de admiração a estadunidenses se reflete em favorecimentos em julgamentos relativos a decisões que impactam a inserção e a progressão desses profissionais no mercado laboral, e a características pessoais que poderiam ser úteis para explicar esses favorecimentos.

\section{Formulação de hipóteses}

A seguir, apresentam-se as hipóteses a serem testadas no estudo, assim como suas respectivas fundamentações teórico-empíricas. As hipóteses encontram-se divididas em dois grupos: as relacionadas a decisões de emprego (avaliação de cumprimento dos requisitos do cargo, contratação e promoção) e aquelas que se remetem às características dos candidatos (competência e cordialidade).

\section{Hipóteses relativas a decisões de emprego}

Diversos estudos têm apresentado evidências de que estrangeiros provenientes de países com menor reputação econômico-social recebem julgamentos menos favoráveis no que se refere às principais decisões de emprego em processos seletivos (Hosoda et al., 2012; Lev-Ari \& Keysar, 2010). Seguindo a mesma lógica, outros estudos (Cargile, 2000; Hosoda \& Stone-Romero, 2010) têm identificado que indivíduos com sotaques considerados representativos de grupos sociais que gozam de reputação positiva no país de destino têm obtido mais avaliações favoráveis em termos de decisões relacionadas a emprego em experimentos sobre a avaliação de estrangeiros por selecionadores a partir do sotaque.

Considerando tanto o sentimento de admiração do brasileiro pelo estadunidense quanto a supervalorização na associação de seu sotaque (Hosoda \& Stone-Romero, 2010), é possível sugerir que esse estrangeiro, ao falar a língua portuguesa em uma disputa de emprego, teria uma vantagem em relação a um concorrente brasileiro se a decisão fosse tomada também por brasileiros. Essa sugestão encontra-se em linha com a evidência de que há, sob a ótica de expatriados provenientes dos Estados Unidos em missão internacional no Brasil, favorecimentos de brasileiros a estadunidenses no que se refere a relações de trabalho (Araujo, Teixeira, \& Malini, 2013). Sendo assim, propõe-se que:

H1: Em comparação a um candidato a uma vaga de trabalho com um sotaque brasileiro no idioma português, um candidato com um sotaque estadunidense no idioma português será mais bem avaliado para uma vaga de Analista de Recursos Humanos em termos de cumprimento dos requisitos do cargo (H1a), probabilidade de recebimento de promoção (H1b) e probabilidade de contratação (H1c).

\section{Hipóteses relativas a características dos candidatos}

Pesquisas anteriores têm encontrado evidências de que pessoas com sotaques considerados como associados a grupos ou indivíduos de um país menos desejados socialmente em outro tendem a ser avaliadas como menos competentes, contudo mais cordiais, quando buscam vagas de trabalhos em países no qual não se fala sua língua materna (Cargile \& Bradac, 2001; Giles et al., 1995; Lee \& Fiske, 2006). Por outro lado, aqueles que apresentam um sotaque que remete a grupos sociais que possuem elevado status ou reputação em um país distinto tendem a ser vistos como pessoas mais inteligentes e competentes quanto indivíduos locais em países considerados social e economicamente dominantes 
(Giles et al., 1995). No entanto, características como bondade, solidariedade e atratividade tendem a ser mais associadas a indivíduos que apresentam sotaques que remetem a grupos não dominantes, como latinos (Cargile \& Bradac, 2001; Sebastian, Ryan, Keogh, \& Schmidt, 1980).

No contexto brasileiro, estudos têm sugerido que indivíduos provenientes de países considerados economicamente mais desenvolvidos, como os Estados Unidos, gozam de uma reputação social positiva entre brasileiros (Araujo et al., 2014). Por outro lado, notam-se também evidências de que é comum que o brasileiro veja seus conterrâneos como pessoas menos aptas a desenvolver atividades que exigem maior competência (Araujo et al., 2013), embora frequentemente se vejam como pessoas com quem se pode desenvolver relacionamentos amistosos e agradáveis (Calligaris, 1991). Aplicando as premissas de estigma presentes nos estudos anteriores que têm identificado relações entre sotaque e avaliações de candidatos à fixação do brasileiro na figura do estadunidense e o desprezo por outros brasileiros, propõese que:

H2: Em comparação a um candidato a uma vaga de trabalho com um sotaque brasileiro no idioma português, um candidato com um sotaque estadunidense no idioma português será mais bem avaliado para uma vaga de Analista de Recursos Humanos em termos de competência (H2a) e mais mal avaliado em termos de cordialidade $(\mathrm{H} 2 \mathrm{~b})$.

\section{Metodologia}

O presente estudo foi realizado sob uma abordagem quantitativa e sob um design de experimento. Essa escolha se deu pelo fato de que as hipóteses retratam um teste em que dois grupos distintos são comparados em termos de critérios específicos, o que pode ser melhor realizado por meio de um design experimento do que por um desenho correlacional. Além disso, como em abordagens experimentais de pesquisa, os participantes não identificam o que está sendo avaliado e manipulado, supõe-se que essa abordagem ajuda a minimizar efeitos de desejabilidade social frequentemente encontrados em estudos correlacionais (H. T. Reis \& Gosling, 2010; Melo \& Farias, 2014).

Para a escolha da amostra, optou-se em buscar avançar em relação a estudos anteriores que apresentaram como limitação o uso de estudantes de graduação como avaliadores (p.e., Deprez-Sims \& Morris, 2010; Hosoda et al., 2012; Hosoda \& Stone-Romero, 2010; Lev-Ari \& Keysar, 2010; Singer \& Eder, 1989). Buscou-se acessar uma amostra em que os participantes tivessem uma maior aproximação da atividade de seleção de pessoal e que apresentassem um nível de qualificação satisfatório. Sendo assim, adotou-se como critério para participação no estudo estar cursando ou ter concluído um curso de MBA em Gestão de Pessoas. A escolha da instituição que possibilitou o acesso aos alunos se deu em função do tamanho do banco de alunos disponibilizado, uma vez que se fazia necessário acessar uma amostra substantiva.

Os participantes do estudo foram divididos aleatoriamente em dois grupos e foram submetidos a uma sessão experimental. Ao início de cada sessão experimental, um pesquisador solicitava ao participante que este: (a) se imaginasse como selecionador de um candidato a uma vaga de trabalho; (b) lesse uma descrição do perfil desejado para uma vaga de Analista de Recursos Humanos, para a qual não havia o requisito de domínio de outros idiomas, além do português; (c) escutasse atentamente uma gravação de áudio na qual um candidato falaria de si por um minuto; e (d) avaliasse o candidato em questão segundo os seguintes critérios: cumprimento dos requisitos do cargo, probabilidade de contratação, probabilidade de recebimento de promoção, competência e cordialidade. $O$ texto da gravação foi adaptado a partir do utilizado em Hosoda et al. (2012) e se encontra no Apêndice A.

Cabe aqui uma explicação: optou-se por realizar o experimento com base em uma vaga de Analista de Recursos Humanos em que não havia a necessidade de fluência em outros idiomas além do português, de forma que o domínio nativo do idioma inglês do candidato fictício com o sotaque estadunidense não representasse uma vantagem competitiva para o cargo em questão na simulação. 
Os indivíduos alocados nos dois grupos foram submetidos a duas distintas condições experimentais: enquanto os membros de um grupo escutaram uma gravação da apresentação de um brasileiro falando com sotaque português paulista, os membros do outro grupo escutaram a gravação de um candidato apresentando-se em português, porém com sotaque estadunidense. A manipulação dos sotaques seguiu o critério sugerido por Lambert (1967): foram realizadas a partir de um mesmo texto lido por um mesmo indivíduo, um ator brasileiro bilíngue (português/ inglês estadunidense). A escolha do uso de um ator bilíngue se deve ao treinamento dele para emular os sotaques. O uso do mesmo texto para ambas as gravações permite que as características paralinguísticas da voz do narrador se mantenham relativamente constantes (Bresnahan, Ohashi, Nebashi, Liu, \& Shearman, 2002). Foi solicitado que o ator memorizasse o texto narrado e o verbalizasse de forma natural e fluida, de forma a aparentar espontaneidade. Após diversas tentativas de gravação, foi escolhida a narração considerada como a mais representativa de cada um dos dois sotaques emulados, e ambas as gravações foram apresentadas para cinco linguistas bilíngues (português/ inglês estadunidense), que não identificaram tratar-se de uma simulação.

Todas as variáveis avaliadas no estudo foram operacionalizadas por meio de uma versão em português de escalas encontradas em Hosoda et al. (2012). As escalas passaram por um processo de tradução reversa para o português. A variável Cumprimento dos Requisitos do Cargo foi medida por meio de três itens (exemplo: Eu acho que ele serve para o cargo) em uma escala de 7 pontos cujas opções de resposta variaram de 1 (discordo fortemente) a 7 (concordo fortemente). Por sua vez, Probabilidade de Recebimento de Promoção foi medida por meio de uma escala de dois itens (exemplo: O potencial de que ele seja promovido nos próximos cinco anos é...) em uma escala de 7 pontos, que varia de 1 (muito baixo) a 7 (muito alta). Já Probabilidade de Contratação foi operacionalizada por meio do item - Você o contrataria para o cargo? - que foi pontuado como 1 para não e 2 para sim. As variáveis Competência Percebida e Cordialidade Percebida, que se referiam às características pessoais dos participantes, foram medidas por meio de escalas de 7 itens para cada. Nessas escalas, foram apresentados adjetivos antônimos (por exemplo: inteligente/não inteligente, confiante/não confiante e competente/incompetente para Competência Percebida; e cordial/frio, amigável/não amigável e honesto/desonesto para Cordialidade Percebida).

Um item adicional foi criado para avaliar a efetividade da manipulação do sotaque estadunidense de um dos candidatos hipotéticos. Foi solicitado que os participantes avaliassem o grau do sotaque do candidato em uma escala Likert de 7 pontos, que variou de 1 (sem sotaque estrangeiro) a 7 (sotaque estrangeiro muito forte).

O questionário utilizado no experimento foi adaptado do utilizado em Hosoda et al. (2012) e encontra-se no Apêndice B.

\section{Resultados}

Neste tópico, apresentam-se o perfil dos participantes do estudo, as análises da avaliação da manipulação do sotaque, as análises preliminares realizadas de forma a verificar a possível relação entre as variáveis demográficas gênero, etnia e idade, e as medidas obtidas em termos de emprego e contratação, e os testes das hipóteses.

\section{Perfil dos participantes do estudo}

O estudo foi realizado com um grupo de 304 alunos e ex-alunos (185 mulheres e 119 homens) de um curso de MBA em Gestão de Pessoas de uma instituição de ensino superior sediada na cidade de São Paulo. Em média, a idade dos participantes era de 29 anos (desvio padrão = 2,82), variando entre as idades de 23 a 53 anos. Um total de $46(15,1 \%)$ participantes tem até 25 anos, outros $121(39,8 \%)$ estão na faixa etária entre 26 e 30 anos, 62 (20,4\%) se classificaram entre 31 e 35 anos, 44 (14,5\%) entre 36 e 40 anos e um total de 31 (10,2\%) participantes possui mais que 40 anos. Em termos de etnia, a amostra 
apresentou a seguinte caracterização: $50,7 \%$ se declararam brancos $(n=154) ; 33,6 \%$ pardos $(n=102)$; $11,8 \%$ negros $(n=36) ; 3,3 \%$ asiáticos $(n=10)$; e $0,7 \%(n=2)$ se classificaram como de etnia indígena. A maioria dos participantes encontrava-se empregado no momento em que participaram da pesquisa $(95,1 \%, n=289)$, já trabalharam na área de Gestão de Pessoas $(92,8 \%, n=282)$, e diversos deles já desempenharam atividades de Seleção de Pessoal $(54,3 \%, n=165)$. Os dados de caracterização da amostra se encontram sumarizados na Tabela 1.

Tabela 1

\section{Caracterização da Amostra}

\begin{tabular}{lcc} 
& \multicolumn{2}{c}{ Frequência } \\
\cline { 2 - 3 } & Absoluta (n) & Percentual (\%) \\
\hline Sexo & 185 & 61 \\
Feminino & 119 & 39 \\
Masculino & & 15,1 \\
\hline Faixa etária & 46 & 39,8 \\
\hline Até 25 & 121 & 20,40 \\
$25-30$ & 62 & 14,50 \\
$31-35$ & 44 & 10,20 \\
$36-40$ & 31 & 50,7 \\
$>40$ & & 33,6 \\
\hline Etnia & 154 & 11,8 \\
\hline Brancos & 102 & 3,3 \\
Pardos & 36 & 0,7 \\
Negros & 10 & \\
Asiáticos & 2 & 95,1 \\
Indígenas & & 92,8 \\
\hline Situação de emprego & 289 & 54,3 \\
\hline Empregado & 282 & \\
Atuando na área de gestão de pessoas & 165 & \\
Atuando em atividades de seleção de pessoal & & \\
\hline
\end{tabular}

Nota. Fonte: Elaborada pelos autores.

\section{Análise de checagem da manipulação do sotaque}

Foi realizada uma ANOVA para checar a efetividade da manipulação do sotaque contido nas gravações utilizadas no experimento. Os resultados indicam que a manipulação foi feita com sucesso: a gravação representativa do candidato com sotaque estadunidense foi avaliada como possuindo um sotaque mais forte $(M=6,48, S D=1,09)$ que a gravação que representava o sotaque brasileiro $(M=2,23$, $\mathrm{SD}=1,63), \mathrm{F}(1,303)=138,76, \mathrm{p}<0,001$. Esse resultado possibilitou qualificar as gravações como adequadas para uso no experimento realizado nesta pesquisa. 


\section{Análises preliminares}

Uma vez que a amostra foi constituída por 185 mulheres e 119 homens, optou-se por examinar se as medidas avaliadas (cumprimento dos requisitos do cargo, probabilidade de contratação, probabilidade de recebimento de promoção, competência e cordialidade percebidas) foram influenciadas pelo efeito de interação entre o gênero do avaliador versus o sotaque do candidato. O resultado de uma MANCOVA 2 (sotaque do candidato) x 2 (gênero do avaliador) mostrou que o gênero dos participantes não apresentou relação com as medidas avaliadas $-\mathrm{F}(5,246)=1,07, \mathrm{p}=0,23, \lambda$ de Wilks $=0,97$ e não mostrou relação com o sotaque do candidato, $\mathrm{F}(5,246)=1,03$, $\mathrm{p}=0,34, \lambda$ de Wilks $=0,98$.

A amostra também se mostrou heterogênea em termos de etnia. Dado esse fato, optou-se por analisar se essa diversidade étnica da amostra teve alguma influência nos resultados das medidas avaliadas, por meio de um teste do efeito de interação entre a etnia dos participantes versus sotaque do candidato. A etnia dos participantes foi dividida em brancos, pardos, negros e outros (asiáticos e indígenas foram agrupados, devido ao pequeno número de participantes que se incluíram nessas categorias). O resultado da MANCOVA 2 (sotaque do candidato) x 4 (etnia do avaliador) realizada mostrou que a etnia dos participantes não produziu efeitos nas medidas avaliadas, $\mathrm{F}(15,462,32)=1,14$, $\mathrm{p}=0,31, \lambda$ de Wilks $=0,94$, nem no sotaque do candidato, $\mathrm{F}(15,462,32)=0,83 \mathrm{p}=0,53, \lambda$ de Wilks $=$ 0,92 .

Com base nesses resultados preliminares, tomou-se a decisão de que todas as hipóteses deveriam ser testadas usando o sotaque do participante como única variável independente do estudo.

\section{Testes das hipóteses}

Na Tabela 2, apresentam-se as estatísticas descritivas e as correlações entre as variáveis do estudo.

Tabela 2

\section{Médias, Desvios-Padrão e Correlações entre as Variáveis Mensuradas}

\begin{tabular}{lccccccc}
\hline Medida & Média & $\begin{array}{c}\text { Desvio } \\
\text { Padrão }\end{array}$ & $\mathbf{1}$ & $\mathbf{2}$ & $\mathbf{3}$ & $\mathbf{4}$ & $\mathbf{5}$ \\
\hline 1- Sotaque do participante & - & - & & & & & \\
2- Cumprimento dos requisitos do cargo & 4,35 & 1,47 & $0,30^{* *}$ & & & & \\
3- Probabilidade de promoção & 4,02 & 1,42 & $-0,2^{* *}$ & 0,12 & & & \\
4- Probabilidade de contratação & 1,48 & 0,48 & $0,28^{* *}$ & $0,45^{* * *}$ & 0,09 & & \\
5- Competência percebida & 4,42 & 1,17 & $0,25^{* *}$ & $0,21^{* *}$ & 0,03 & $0,35^{* *}$ & \\
6- Cordialidade percebida & 3,92 & 0,97 & $-0,3^{* *}$ & $-0,1^{* *}$ & $0,36^{* * *}$ & $-0,2^{* *}$ & $0,19^{*}$ \\
\hline
\end{tabular}

Nota. Fonte: Elaborada pelos autores.

$* \mathrm{p}<0,05 ; * * \mathrm{p}<0,01 ; * * * \mathrm{p}<0,00$.

Os resultados apresentados mostram que o sotaque dos participantes apresentou uma relação com todas as variáveis mensuradas (Cumprimento dos requisitos do cargo, Probabilidade de recebimento de promoção, Probabilidade de contratação, Competência percebida e Cordialidade percebida), embora algumas dessas relações tenham sido negativas (com Probabilidade de promoção e Cordialidade percebida), ao contrário do intuído na argumentação que sustenta a elaboração deste estudo. Após a apresentação desses coeficientes, os quais possibilitam uma visão geral dos resultados do estudo, foram realizados os testes das hipóteses elaboradas. A seguir apresentam-se os resultados encontrados, organizados de acordo com dois blocos de hipóteses: primeiro, apresentam-se os resultados relacionados às hipóteses $1 \mathrm{a}, 1 \mathrm{~b}$ e $1 \mathrm{c}$ (referentes a decisões de emprego) e, em seguida, os resultados dos testes das hipóteses $2 \mathrm{a}$ e $2 \mathrm{~b}$ (referentes a características pessoais dos candidatos). 


\section{Hipóteses referentes a decisões de emprego}

O primeiro grupo de hipóteses apresentadas no presente estudo se refere a decisões de emprego e defendem que, em comparação a um candidato a uma vaga de trabalho com um sotaque brasileiro no idioma português, um candidato com um sotaque estadunidense no idioma português será mais bem avaliado para uma vaga de Analista de Recursos Humanos em termos de cumprimento dos requisitos do cargo (H1a), probabilidade de recebimento de promoção (H1b) e probabilidade de contratação (H1c). Para testar essas hipóteses, realizou-se uma análise de variância multivariada (One-way MANOVA) entre participantes. Os resultados mostraram um efeito do sotaque dos candidatos, $\mathrm{F}(2,302)=8,32$, $\mathrm{p}<0,01, \lambda$ de Wilks $=0,90$.

Uma subsequente ANOVA mostrou que o sotaque dos candidatos apresentou relação com o Cumprimento dos requisitos do cargo, $\mathrm{F}(1,303)=11,12$, $\mathrm{p}<0,01$, e com a Probabilidade de Contratação, $\mathrm{F}(1,303)=13,06, \mathrm{p}<0,01$. As avaliações da gravação em áudio com sotaque estadunidense foram mais positivas em termos de cumprimento dos requisitos do cargo $(\mathrm{M}=4,71, \mathrm{SD}$ $=1,51)$ do que as avaliações referentes ao candidato com o sotaque brasileiro $(\mathrm{M}=4,01, \mathrm{SD}=1,36)$, $\mathrm{o}$ que não permite rejeitar a H1a. Diferentemente do hipotetizado neste estudo, o sotaque estadunidense foi pior avaliado em termos de Probabilidade de Promoção $(\mathrm{M}=3,80, \mathrm{SD}=1,32)$ em relação às avaliações referentes ao sotaque brasileiro $(\mathrm{M}=4,24, \mathrm{SD}=1,43)$, o que leva à rejeição da $\mathrm{H} 1 \mathrm{~b}$.

Como a variável Probabilidade de Contratação foi mensurada como categórica (opções de resposta não e sim, conforme descrito na metodologia), a H1c foi testada por meio de um teste $\chi 2$. Os resultados mostram que o sotaque do candidato influenciou a decisão de contratação, $\chi 2(\mathrm{n}=203)=$ $9,62, \mathrm{p}<0,01$. Como apresentado na Tabela 3, enquanto a maioria dos participantes do estudo que escutaram a gravação com o sotaque brasileiro responderam que não contratariam o candidato ( $\mathrm{n}=90$, $59,2 \%$ ), a maioria dos que escutaram a gravação com o sotaque estadunidense responderam que o contratariam $(n=83,54,3 \%)$. Em conjunto, esses resultados não permitem a rejeição de H1c.

Tabela 3

Decisão de Contratação por Tipo de Sotaque no Idioma Português

\begin{tabular}{lcc}
\cline { 2 - 3 } & \multicolumn{2}{c}{ Você contraria esse candidato? } \\
\hline Sotaque & Não & Sim \\
Português-Brasileiro & 90 & 62 \\
Português-Estadunidense & 69 & 83 \\
\hline
\end{tabular}

Nota. Fonte: Elaborada pelos autores.

\section{Hipóteses referentes a características pessoais dos candidatos}

Neste estudo, foi hipotetizado que, em comparação a um candidato a uma vaga de trabalho com um sotaque brasileiro no idioma português, um candidato com um sotaque estadunidense no idioma português seria melhor avaliado para uma vaga de Analista de Recursos Humanos em termos de competência (H2a) e pior avaliado em termos de cordialidade (H2b). Essas hipóteses foram testadas por meio de uma análise de variância multivariada (One-way MANOVA) entre participantes. Os resultados indicam um efeito do sotaque dos candidatos, $\mathrm{F}(2,302)=9,32, \mathrm{p}<0,01, \lambda$ de Wilks $=0,92$.

Em seguida, foi realizada uma ANOVA para melhor explorar esses resultados, e esta indicou que o sotaque dos candidatos mostrou uma relação com a Competência percebida, $\mathrm{F}(1,303)=10,73$, $\mathrm{p}<0,01$, e com a Cordialidade percebida, $\mathrm{F}(1,303)=14,39, \mathrm{p}<0,01$. $\mathrm{O}$ candidato representado na gravação em áudio com sotaque estadunidense foi avaliado como mais competente $(\mathrm{M}=4,61, \mathrm{SD}=$ $1,11)$ do que o candidato representado na gravação com sotaque brasileiro $(\mathrm{M}=4,23, \mathrm{SD}=1,21)$, o que não permite rejeitar a H2a. Conforme hipotetizado, o sotaque estadunidense foi pior avaliado em termos 
de Cordialidade $(\mathrm{M}=3,74, \mathrm{SD}=1,07)$ em relação às avaliações referentes ao sotaque brasileiro $(\mathrm{M}=$ $4,10, \mathrm{SD}=1,16)$, o que também não permite rejeitar $\mathrm{H} 2 \mathrm{~b}$.

\section{Discussão}

Apesar da presença crescente de profissionais provenientes de economias dominantes em países emergentes, há uma forte necessidade de explorar a influência do sotaque de estrangeiros em decisões de emprego no Brasil. A partir dessa lacuna de pesquisa, este estudo objetivou examinar a relação entre o sotaque de um candidato a uma vaga de trabalho hipotética e julgamentos relacionados ao emprego e à contratação. $\mathrm{O}$ estudo focou especificamente os sotaques brasileiro e estadunidense no idioma português. A seguir, realiza-se um confronto entre os achados da pesquisa e a literatura prévia, de forma a salientar pontos em que o argumento derivado no referencial teórico foi evidenciado e aspectos em que os resultados permitem expandir um dos pontos do argumento desta pesquisa: o de que a estigmatização a estadunidenses em processos seletivos no Brasil dar-se-ia por favorecimentos, quando comparados a um candidato equivalente brasileiro.

\section{Discussão referente aos julgamentos relacionados a decisões de emprego}

Os resultados sugerem que, em comparação a um candidato brasileiro em um processo seletivo, um indivíduo com sotaque estadunidense estaria em vantagem em termos de chances de contratação para um cargo de Analista de Recursos Humanos. Além disso, o candidato com sotaque estadunidense foi visto como estando mais apto ao cargo. Um resultado que pode ser útil para interpretar esses achados se refere ao fato de que o indivíduo representado na gravação feita com um sotaque estadunidense foi também avaliado como mais competente. Pesquisas anteriores apresentaram uma relação positiva entre competência e cumprimento dos requisitos do cargo (Hosoda et al., 2012), o que configura uma consistência entre os resultados deste estudo e a literatura recente sobre o tema. Este resultado, como esperado, difere dos geralmente encontrados em estudos sobre a influência de sotaque de estrangeiros em países dominantes economicamente (Hosoda et al., 2012; Lev-Ari \& Keysar, 2010; Singer \& Eder, 1989). Conforme sugerido por Araujo, Teixeira, Cruz e Malini (2014), o traço cultural brasileiro estrangeirismo parece influenciar a atitude em relação a profissionais provenientes dos Estados Unidos, país que hoje ocupa a posição de principal referência econômica e cultural para o brasileiro (Motta $e t$ al., 2001).

Porém, apesar de ser avaliado como mais apto ao cargo e com maior chance de contratação, o candidato hipotético com sotaque estadunidense apresentou menor probabilidade de ser promovido. Esse foi um resultado não esperado encontrado na pesquisa, e que permite reflexões visando a confrontações teóricas contraintuitivas e não previsíveis. O resultado sugere que o estrangeirismo do brasileiro pode não representar apenas benefícios ao profissional oriundo de países dominantes. Uma explicação possível para esse resultado se deve ao fato de que o candidato hipotético com sotaque estadunidense foi avaliado como menos cordial, o que, em uma cultura que privilegia a harmonia entre os relacionamentos como a brasileira (Holanda, 1995), pode representar uma propensão negativa a se submeter à subordinação de indivíduos menos amistosos. Sendo assim, essa evidência empírica fornece subsídios para a contestação da visão monolítica encontrada em Calligaris (1991) e contestada por Araujo, Teixeira, Malini (2013), de que o estrangeirismo seria um traço unidimensional, que se restringiria a uma relação de favorecimentos a estrangeiros de países social e economicamente dominantes.

Interpretados de forma conjunta, os resultados referentes aos julgamentos em decisões de empregos aqui investigados revelam uma relação profissional complexa do brasileiro em relação ao americano. Diferentemente do encontrado na literatura sobre estrangeirismo, que tende a interpretar tal relação como unidimensional e positiva, os resultados aqui demonstrados sugerem que o julgamento positivo em relação ao estrangeiro possa ser função do tipo de relação que se tem com esse estrangeiro, 
de forma que o favorecimento tenderia a ocorrer, para o cargo descrito, em casos de contratação, mas não de promoção.

\section{Discussão referente aos atributos pessoais dos candidatos}

Os resultados encontrados referentes aos atributos pessoais de competência e cordialidade percebidas, por sua vez, alinharam-se com a literatura sobre atitudes em relação a línguas. O candidato com sotaque estadunidense no idioma português foi visto como mais competente e menos cordial que o indivíduo com sotaque brasileiro. Tais achados são consistentes com os encontrados por Hosoda et al. (2012) e de Lee e Fiske (2006), nos quais os latinos (mais especificamente sul-americanos e mexicanos) foram percebidos também como menos competentes e mais cordiais que os estadunidenses. No entanto, nos citados estudos, a avaliação foi realizada por estadunidenses, enquanto no presente trabalho ela foi realizada por latinos (brasileiros). Com isso, pode-se dizer que esse resultado representa um avanço para a literatura sobre atitudes em relação a línguas, ao encontrar um padrão similar de percepção de atributos pessoais de estadunidenses e latinos segundo a avaliação de indivíduos de ambas as culturas. Esse resultado coaduna com a ideia de que sotaques possuem um valor simbólico (Lev-Ari \& Keysar, 2010), visto que se têm notado percepções distintas em termos de competência e cordialidade de acordo com o nível socioeconômico dos países aos quais os sotaques estão associados.

De forma geral, o estudo mostrou diferenças entre os julgamentos realizados em relação aos candidatos hipotéticos. Dado que a voz e o texto a partir dos quais as gravações foram realizadas e que o perfil demográfico dos membros dos dois grupos de participantes era idêntico, as diferenças encontradas em todos os critérios avaliados sinalizam para a presença de vieses nos julgamentos. Os resultados, no entanto, não sinalizaram uma avaliação estigmatizada e desfavorável do grupo dominado em todas as dimensões avaliadas, como se poderia esperar, com base em Cargile (2000).

\section{Considerações Finais}

O estudo realizado apresenta algumas limitações, as quais se buscam apresentar neste tópico, acompanhadas de direções para pesquisas a serem realizadas. Primeiro, aponta-se o fato de que os avaliadores do experimento realizado não são necessariamente tomadores de decisão, o que poderia gerar vieses em termos da similaridade das decisões tomadas pelos participantes da pesquisa em relação às decisões que seriam tomadas na vida real. Todavia, neste estudo, buscou-se minimizar esse problema ao utilizar uma amostra de alunos de MBA em um curso em que os alunos apresentam maior familiaridade com a prática de seleção, em vez de adotar a recorrente prática de contar com avaliações realizadas por alunos de graduação. De qualquer forma, sugere-se que futuros estudos realizados com pessoas em cargo de tomada de decisão de contratação e promoção em empresas permitam avaliar a validade externa dos resultados encontrados.

Segundo, a avaliação realizada pelos participantes da pesquisa teve como parâmetro as características de um cargo de Analista de Recursos Humanos. Desta forma, não é possível afirmar que os resultados encontrados reflitam uma tendência de julgamento do brasileiro em relação a compatriotas e estadunidenses de forma ampla. Recomenda-se que futuras pesquisas explorem cargos com diferentes graus de status social, de forma a melhor compreender como os julgamentos de brasileiros em relação a profissionais americanos em termos de contratação e promoção podem variar de acordo com o cargo em questão.

Terceiro, o estudo foi realizado a partir da gravação de um indivíduo com um sotaque paulista para o idioma português, com avaliadores residentes na cidade de São Paulo. Estudos comparativos poderiam ser realizados com avaliadores de outras capitais do país onde haja uma alta concentração de profissionais estadunidenses. Essa oportunidade de pesquisa permitiria um melhor entendimento a respeito das variações existentes dentro do país, no que se refere a possíveis favorecimentos ou 
preconceitos a estadunidenses. Tal abordagem seria especialmente relevante no caso brasileiro devido às dimensões continentais e à diversidade cultural características do país.

Quarto, o estudo se limita a avaliar o julgamento referente a um candidato estadunidense. Devido ao crescimento no número de estrangeiros de diferentes origens trabalhando no Brasil (Araujo et al., 2014), seria oportuno replicar este estudo utilizando gravações que simulem outros sotaques, como o espanhol. Além disso, comparações intranacionais (Deshields \& Kara, 2011; Rakic, Steffens, \& Mummendey, 2011) que simulem sotaques de diferentes regiões do Brasil e que remetam a distintas ISVs poderiam oferecer uma leitura útil para avanços teóricos no tocante ao entendimento quanto aos preconceitos e privilégios de brasileiros de diferentes regiões no contexto do trabalho.

Quinto, a única variável manipulada no estudo foi o sotaque. Contudo, outras variáveis poderão ser gerenciadas em futuros estudos, como o nome e o gênero do candidato. Purkiss, Perrewe, Gillespie, Mayes e Ferris (2006) e Hosoda et al. (2012) sugerem que manipulações simultâneas de diferentes variáveis que podem interferir em julgamentos de contratação podem revelar efeitos sinérgicos em termos de avaliações favoráveis ou desfavoráveis aos candidatos em questão. Em outras palavras, endossa-se a sugestão de que futuros estudos manipulem não somente o sotaque, mas também o nome do candidato, de forma a avaliar se a presença ou ausência de dois sinais que podem remeter a ISVs (in)desejadas potencializariam o grau de explicação das variáveis de julgamento incluídas no estudo. $\mathrm{O}$ gênero pode ser manipulado em futuras pesquisas por meio da apresentação de currículos com conteúdos iguais, com exceção do gênero do candidato, a dois grupos. Neste caso, o efeito de sinergia com o sotaque não poderia ser testado dado que a gravação de vozes masculina e feminina não poderiam ser feitas pelo mesmo ator.

Finalmente, a abordagem quantitativa do estudo limita os resultados a um caráter descritivo estatístico. Pesquisas adicionais de natureza qualitativa poderiam melhor explorar os porquês de a avaliação referente à gravação de um candidato hipotético com sotaque estadunidense ter refletido uma tendência a considerar o estadunidense em termos de contratação, mas não de promoção. Sendo assim, sugere-se que sejam estudados os significados atribuídos por brasileiros à ISV de trabalhadores estadunidenses, conferindo especial atenção aos atributos pessoais de competência e cordialidade.

Em termos práticos, sugere-se que organizações busquem contratar selecionadores mais capazes de manifestar maior neutralidade no que se refere a avaliações profissionais de indivíduos com sotaques estrangeiros. No caso aqui estudado, os resultados apontam que, em relações de trabalho desenvolvidas no contexto brasileiro, trabalhadores estadunidenses tendem a obter vantagem sobre brasileiros na contratação e na desvantagem em termos de promoção, em razão de seu sotaque. Essa tendência é resultado de um processo irracional e enviesado que pode levar as organizações a adotarem decisões que não promovem os interesses organizacionais de maximização de resultado. $\mathrm{O}$ fato de que diversas empresas têm realizado etapas intermediárias do processo seletivo por telefone, e que muitos trabalham em postos de trabalho remoto e só possuem contato com os profissionais da área de Gestão de Pessoas da empresa por telefone e/ou e-mails potencializa os riscos do viés aqui apontado.

Cabe ressaltar a limitação de o estudo não haver sido realizado exclusivamente com profissionais que atuam em função de seleção de pessoas, já que isso não permite dizer de maneira assertiva que os resultados refletem a prática desses profissionais. No entanto, com base na mesma lógica de estudos anteriores sobre o tema realizados com estudantes de graduação (Deprez-Sims \& Morris, 2010; Hosoda et al., 2012; Hosoda \& Stone-Romero, 2010; Singer \& Eder, 1989), entendemos que o fato de a amostra não ser constituída integralmente por selecionadores não impede a realização de sugestões para a prática, dado que a estigmatização é um fenômeno social de penetração ampla (Goffman, 1963). De qualquer forma, como a amostra utilizada neste estudo foi composta por profissionais em nível de especialização em Gestão de Pessoas, os quais predominantemente atuam na área, geralmente na função de selecionadores de pessoal, entende-se que, se por um lado, há limitações para a realização de sugestões para a prática, por outro, há um avanço em relação à literatura empírica sobre o tema no que tange à similaridade entre a amostra e a população. Isso reforça a adequação das sugestões práticas deste estudo vis-à-vis o estado de desenvolvimento do campo de pesquisas em que esta se insere. 
De forma geral, o estudo destaca a importância das características do discurso não relacionadas ao conteúdo, especificamente o sotaque estrangeiro, em decisões de emprego. Os vieses encontrados nesse processo sugerem que há implicações em termos de oportunidades de ganhos financeiros e de progressão de carreira para indivíduos estrangeiros no Brasil. Sendo assim, espera-se que as organizações desenvolvam políticas de Gestão de Pessoas que venham a neutralizar esse efeito não somente em processos de decisão de contratação e promoção, mas também em todo o sistema de relações humanas no trabalho. Apesar de haver uma corrente defensora de que empresas devam realizar treinamentos para a neutralização de sotaques mesmo para indivíduos que já falam o idioma do país em que trabalham (Rajini, 2009), entende-se aqui que a alteração a ser feita reside no campo organizacinal, e não no comportamento do estrangeiro.

\section{Referências}

Almeida, S., Fernando, M., \& Sheridan, A. (2012). Revealing the screening: organisational factors influencing the recruitment of immigrant professionals. The International Journal of Human Resource Management, 23(9), 1950-1965. http://dx.doi.org/10.1080/09585192.2011.616527

Araujo, B. F. von B., Teixeira, M. L. M., Cruz, P. B. da, \& Malini, E. (2014). Understanding the adaptation of organizational and self-initiated expatriates in the context of Brazilian culture. The International Journal of Human Resource Management, 25(18), 2489-2509. http://dx.doi.org/10.1080/09585192.2012.743470

Araujo, B. F. von B., Teixeira, M. L. M., \& Malini, E. (2013). Estrangeirismo e complexo de Gulliver: brasileiros na percepção de expatriados de diferentes origens. Organizações \& Sociedade, 20(66), 461-478. http://dx.doi.org/10.1590/S1984-92302013000300006

Bandeira, M. (2004). As relações perigosas: Brasil - Estados Unidos (de Collor a Lula, 1990-2004). Rio de Janeiro: Civilização Brasileira.

Bresnahan, M. J., Ohashi, R., Nebashi, R., Liu, W. Y., \& Shearman, S. M. (2002). Attitudinal and affective response toward accented English. Language \& Communication, 22(2), 171-185. http://dx.doi.org/10.1016/s0271-5309(01)00025-8

Brookfield Global Relocation Services. (2012). Global relocation trends: 2012 survey report. Recuperado de https://espritgloballearning.com/wp-content/uploads/2011/03/2012-BrookfieldGlobal-Relocations-Trends-Survey.pdf

Calligaris, C. (1991). Hello Brasil!: notas de um psicanalista europeu viajando ao Brasil. São Paulo: Escuta.

Cargile, A. C. (2000). Evaluation of employment suitability: does accent always matter? Journal of Employment Counseling, 37(3), 165-177. http://dx.doi.org/10.1002/j.2161-1920.2000.tb00483.x

Cargile, A. C., \& Bradac, J. J. (2001). Attitudes toward language: a review of applicant-evaluation research and a general process model. In W. B. Gudykunst (Ed.), Communication yearbook 25 (pp. 347-382). Mahwah, Nj: Lawrence Erlbaum.

Coordenação Geral de Imigração. (2011). Base estatística CGIg: 2. Resumos das autorizações de trabalho temporário. Brasília: Editora Nacional.

Deprez-Sims, A., \& Morris, S. B. (2010). Accents in the workplace: their effects during a job interview. $\begin{array}{llll}\text { International of Journal Psychology, } & \text { 45(6), }\end{array}$ http://dx.doi.org/10.1080/00207594.2010.499950 
Deshields, O. W., \& Kara, A. (2011). The varying influence of spokesperson's accent in communication effectiveness: a comparative study in two different regions of Mexico. Journal of Targeting, Measurement \& Analysis for Marketing, 19(1), 55-65. http://dx.doi.org/10.1057/jt.2011.5

Dubar, C. (2002). La socialisation, construction des identities sociales et profissionnelles. Paris: Armand Colin.

Fazio, L. K., Barber, S. J., Rajaram, S., Ornstein, P. A., \& Marsh, E. J. (2013). Creating illusions of knowledge: learning errors that contradict prior knowledge. Journal of Experimental Psychology: General, 142(1), 1-5. http://dx.doi.org/10.1037/a0028649

Ferguson, M. J., \& Zayas, V. (2009). Automatic evaluation. Current Directions in Psychological Science, 18(6), 362-366. http://dx.doi.org/10.1111/j.1467-8721.2009.01668.x

Gati, I. (1986). Making career decisions: a sequential elimination approach. Journal of Counseling Psychology, 33(4), 408-417. http://dx.doi.org/10.1037/0022-0167.33.4.408

Gellat, H. B. (1962). Decision making: a conceptual frame of reference for counseling. Journal of Counseling Psychology, 9(3), 240-245. http://dx.doi.org/10.1037/h0046720

Giles, H., Willians, A., Mackie, D. M., \& Rosselli, F. (1995). Reactions to anglo- and hispanic-americanaccented speakers: affect, identity, persuasion, and the English-only controversy. Language \& Communication, 15(2), 107-120. http://dx.doi.org/10.1016/0271-5309(94)00019-9

Goffman, E. (1963). Stigma. Notes on the management of spoiled identity. Englewood Cliffs, New Jersey: Prentice-Hall.

Holanda, S. B. (1995). Raízes do Brasil (26a ed.). São Paulo: Companhia das Letras.

Hosoda, M., Nguyen, L. T., \& Stone-Romero, E. F. (2012). The effect of hispanic accents on employment decisions. Journal of Managerial Psychology, 27(4), 347-364. http://dx.doi.org/10.1108/02683941211220162

Hosoda, M., \& Stone-Romero, E. F. (2010). The effects of foreign accents on employment-related decisions. Journal of Managerial Psychology, 25(2), 113-132. http://dx.doi.org/10.1108/02683941011019339

Katz, M. (1966). A model for guidance for career decision making. Vocational Guidance Quarterly, 15(1), 275-288. http://dx.doi.org/10.1002/j.2164-585X.1966.tb01148.x

Lambert, W. E. (1967). A social psychology of bilingualism. Journal of Social Issues, 23(2), 91-109. http://dx.doi.org/10.1111/j.1540-4560.1967.tb00578.x

Lee, T. L., \& Fiske, S. T. (2006). Not an outgroup, not yet an ingroup immigrants in the stereotype content model. International Journal of Intercultural Relations, 30(6), 751-768. http://dx.doi.org/10.1016/j.ijintrel.2006.06.005

Lev-Ari, S., \& Keysar, B. (2010). Why don't we believe non-native speakers? The influence of accent on credibility. Journal of Experimental Social Psychology, 46(6), 1093-1096. http://dx.doi.org/10.1016/j.jesp.2010.05.025

Lick, D. J., \& Johnson, K. L. (2013). Fluency of visual processing explains prejudiced evaluations following categorization of concealable identities. Journal of Experimental Social Psychology, 49(3), 419-425. http://dx.doi.org/10.1016/j.jesp.2013.01.001

Mcglone, M. S., \& Tofighbakhsh, J. (2000). Birds of a feather flock conjointly(?): rhyme as reason in aphorisms. Psychological Science, 11(5), 424-428. http://dx.doi.org/10.1111/1467-9280.00282 
Melo, F. V. S., \& Farias, S. A. de (2014). Sustentabilidade como fator de identidade de destinos turísticos em websites: o consumidor se importa? Brazilian Business Review, 11(2), 143-167. http://dx.doi.org/10.15728/bbr.2014.11.2.6

Motta, F. C. P., Alcadipani, R., \& Bresler, R. B. (2001). A valorização do estrangeiro como segregação nas organizações [Edição Especial]. Revista de Administração Contemporânea, 5, 59-79. http://dx.doi.org/10.1590/S1415-65552001000500004

Murtagh, N. L., Lopes, P. N., \& Lyon, E. (2011). Decision making in voluntary career change: an otherthan-rational perspective. The Career Development Quarterly, 59(3), 249-263. http://dx.doi.org/10.1002/j.2161-0045.2011.tb00067.x

Neumann, J. V., \& Morgenstern, O. (1953). Theory of games and economic behavior. Princeton, NJ: Princeton University Press.

Offermann, L. R., Matos, K., \& Basu DeGraaf, S. (2014). ¿Están hablando de mí?: Challenges for multilingual organizations. Journal of Managerial Psychology, 29(6), 644-660. http://dx.doi.org/10.1108/JMP-10-2012-0315

Oppenheimer, D. M. (2008). The secret life of fluency. Trends in Cognitive Science, 12(6), 237-241. http://dx.doi.org/10.1016/j.tics.2008.02.014

Pudelko, M., Tenzer, H., \& Harzing, A. W. (2014). Cross-cultural management and language studies within international business research: past and present paradigms and suggestions for future research. London: Routledge.

Purkiss, S. K. S., Perrewe, P. K., Gillespie, T. L., Mayes, B. T., \& Ferris, G. R. (2006). Implicit sources of bias in employment interview judgments and decisions. Organizational Behavior and Human Decisions Processes, 101(2), 152-167. http://dx.doi.org/10.1016/j.obhdp.2006.06.005

Rajini, V. (2009). Accent neutralization for effective communication. Iup Journal of Soft Skills, 3(3/4), 45-49.

Rakic, T., Steffens, M. C., \& Mummendey, A. (2011). When it matters how you pronounce it: the influence of regional accents on job interview outcome. British Journal of Psychology, 102(4), 868-883. http://dx.doi.org/10.1111/j.2044-8295.2011.02051.x

Reber, R., \& Schwarz, N. (1999). Effects of perceptual fluency on judgments of truth. Consciousness and Cognition, 8(3), 338-342. http://dx.doi.org/10.1006/ccog.1999.0386

Reber, R., Schwarz, M., \& Winkielman, P. (2004). Processing fluency and aesthetic pleasure: is beauty in the perceiver's processing experience? Personality and Social Psychology Review, 8(4), 364382. http://dx.doi.org/10.1207/s15327957pspr0804_3

Reis, G. G., Fleury, M. T. L., Fleury, A. C. C., \& Zambaldi, F. (2015). Brazilian multinationals' competences: impacts of a "tug of war" between cultural legacies and global $\begin{array}{llll}\text { mindedness. Brazilian } & \text { Business }\end{array}$ http://dx.doi.org/10.15728/bbr.2015.12.1.3

Reis, H. T., \& Gosling, S. D. (2010). Social psychological methods outside the laboratory. In S. T. Fisky, D. T. Gilbert, \& G. Lindzey (Eds.), Handbook of social psychology (pp. 82-114). New York: John Wiley \& Sons.

Schwarz, M., Sanna, L. J., Skurnik, I., \& Yoon, C. (2007). Metacognitive experiences and the intricacies of setting people straight: implications for debiasing and public information campaigns. Advances in Experimental Social Psychology, 39, 127-161. http://dx.doi.org/10.1016/S00652601(06)39003-X 
Schwarz, N. (2004). Meta-cognitive experiences in consumer judgment and decision making. Journal of Consumer Psychology, 14(4), 332-348. http://dx.doi.org/10.1207/s15327663jcp1404_2

Sebastian, R. J., Ryan, E. B., Keogh, T. F., \& Schmidt, A. C. (1980). The effects of negative affect arousal on reactions to speakers. In H. Giles, W. Robinson, \& P. M. Smith (Eds.), Language: social psychological perspectives (pp. 203-208). Pergamon: Oxford.

Simon, H. A. (1955). Behavioral model of rational choice. Quarterly Journal of Economics, 69(1), 99118. http://dx.doi.org/10.2307/1884852

Singer, M., \& Eder, G. S. (1989). Effects of ethnicity, accent, and job status on selection decisions. $\begin{array}{llll}\text { International Journal of } & \text { Psychology, } & \text { 24(1/5), } & \text { 134. }\end{array}$ http://dx.doi.org/10.1080/00207594.1989.10600029

Smith, P. B., Torres, C. V., Leong, C. H., Budhwar, P., Achoui, M., \& Lebedeva, N. (2012). Are indigenous approaches to achieving influence in business organizations distinctive? A comparative study of guanxi, wasta, jeitinho, svyazi and pulling strings. The International Journal of Human Resource Management, 333-348. http://dx.doi.org/10.1080/09585192.2011.561232

Soares, H. F. G., \& Barbedo, C. H. S. (2013). Desempenho acadêmico e a teoria do prospecto: estudo empírico sobre o comportamento decisório. Revista de Administração Contemporânea, 17(1), 6482. Recuperado de http://www.scielo.br/pdf/rac/v17n1/v17n1a05.pdf. http://dx.doi.org/10.1590/S1415-65552013000100005

Song, H., \& Schwarz, N. (2009). If it's difficult to pronounce, it must be risky: fluency, familiarity, and risk perception. Psychological Science, 20(2), 135-138. http://dx.doi.org/10.1111/j.14679280.2009.02267.x

Stone-Romero, E. F., \& Stone, D. L. (2007). Cognitive, affective, and cultural influences on stigmatization: impact on human resource management processes and practices. Research in Personnel and Human Resources Management, 26, 117-67.

Unkelbach, C. (2007). Reversing the truth effect: learning the interpretation of processing fluency in judgments of truth. Journal of Experimental Psychology: Learning, Memory, and Cognition, 33(1), 219-230. http://dx.doi.org/10.1037/0278-7393.33.1.219

\section{Authors' Profiles}

Bruno Felix von Borell de Araujo

Av. Fernando Ferrari, 1358, Boa Vista, 29075-505, Vitória, ES, Brasil. E-mail: bfelix@ fucape.br

Fabricia Correa

Av. Fernando Ferrari, 1358, Boa Vista, 29075-505, Vitória, ES, Brasil. E-mail: briciacorrea@gmail.com

Mark Wolters

350 Wohlers Hall, 1206, South Sixth Street, 61820, Champaign, IL, USA. E-mail: mwolter@illinois.edu 


\section{APÊNDICE A}

\section{Texto usado nas gravações utilizadas no experimento}

Eu possuo uma graduação em Administração e me considero uma pessoa ambiciosa e cheia de motivação. Eu gosto de assumir riscos e nunca desisto de vencer os meus desafios. Eu faço tudo o que está ao meu alcance para atingir meus objetivos, desde que não precise pisar em ninguém. Apesar de eu trabalhar bem em equipe, eu prefiro trabalhar de forma independente. Eu tenho certa facilidade para executar diferentes atividades ao mesmo tempo. Sou uma pessoa colaborativa e acredito que ser colaborativo seja um elemento importante para o sucesso profissional de uma pessoa. Eu confesso que às vezes acabo deixando algumas coisas para a última hora, mas eu lido bem ao trabalhar sob pressão. Eu tenho um desejo muito grande de ser selecionado para essa vaga. 


\section{APÊNDICE B}

\section{Questionário utilizado no experimento}

Cumprimento dos requisitos do cargo

1. ___ Eu acho que ele é adequado para o cargo.

2. E__ Eu acho que ele possui o conhecimento e as habilidades necessárias para desempenhar o cargo.

3. ___ Eu acredito que ele é qualificado para o cargo.

Probabilidade de Recebimento de Promoção

1. O potencial de que ele seja promovido nos próximos cinco anos é:

$\begin{array}{llllllllll}\text { Muito baixo } & 1 & 2 & 3 & 4 & 5 & 6 & 7 & \text { Muito alto }\end{array}$

2. A probabilidade de ele se mover para uma posição de nível gerencial é:

$\begin{array}{llllllllll}\text { Muito baixo } & 1 & 2 & 3 & 4 & 5 & 6 & 7 & \text { Muito alta }\end{array}$

Probabilidade de contratação

1. Você o contrataria para o cargo?

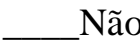

$\operatorname{Sim}$

Competência Percebida

1. Educado

$\begin{array}{lllllll}1 & 2 & 3 & 4 & 5 & 6 & 7\end{array}$

Não educado

2. De classe

$\begin{array}{lllllllll}1 & 2 & 3 & 4 & 5 & 6 & 7 & \text { Sem classe }\end{array}$

3. Inteligente

$\begin{array}{llllllll}1 & 2 & 3 & 4 & 5 & 6 & 7 & \text { Não inteligente }\end{array}$

4. Administrativo

$\begin{array}{llllll}1 & 2 & 3 & 4 & 5 & 6\end{array}$

Operacional

5. Em vantagem

$\begin{array}{lllllll}1 & 2 & 3 & 4 & 5 & 6\end{array}$

Em desvantagem

6. Confiante

$\begin{array}{llllll}1 & 2 & 3 & 4 & 5 & 6\end{array}$

Não confiante

7. Competente

$\begin{array}{llllllll}1 & 2 & 3 & 4 & 5 & 6 & 7 & \text { Incompetente }\end{array}$

Cordialidade Percebida

$\begin{array}{lllllllll}\text { 1. Amável } & 1 & 2 & 3 & 4 & 5 & 6 & 7 & \text { Não amável } \\ \text { 2. Cordial } & 1 & 2 & 3 & 4 & 5 & 6 & 7 & \text { Frio } \\ \text { 3. Amigável } & 1 & 2 & 3 & 4 & 5 & 6 & 7 & \text { Não amigável } \\ \text { 4. Simpático } & 1 & 2 & 3 & 4 & 5 & 6 & 7 & \text { Antipático } \\ \text { 5. Agradável } & 1 & 2 & 3 & 4 & 5 & 6 & 7 & \text { Desagradável } \\ \text { 6. Atencioso } & 1 & 2 & 3 & 4 & 5 & 6 & 7 & \text { Não atencioso }\end{array}$




\section{$\begin{array}{llllllllll}\text { 7. Honesto } & 1 & 2 & 3 & 4 & 5 & 6 & 7 & \text { Desonesto }\end{array}$}

Verificação da manipulação do sotaque

1. No que se refere a possuir sotaque estrangeiro forte, o candidato:

$\begin{array}{llllllllll}\text { Não possui } & 1 & 2 & 3 & 4 & 5 & 6 & 7 & \text { Possui }\end{array}$

2. Para você qual a raça/etnia dele?:

_ Branco _ Pardo _ Negro _- Asiático __ Indígena _ Outro 